\title{
Application Areas of Community Detection:
}

\section{A Review}

\author{
Arzum Karataş \\ Department of Computer Engineering \\ Izmir Institute of Technology \\ İzmir, Turkey \\ arzumkaratas@iyte.edu.tr
}

\author{
Serap Şahin \\ Department of Computer Engineering \\ Izmir Institute of Technology \\ İzmir, Turkey \\ serapsahin@iyte.edu.tr
}

\begin{abstract}
In the realm of today's real world, information systems are represented by complex networks. Complex networks contain a community structure inherently. Community is a set of members strongly connected within members and loosely connected with the rest of the network. Community detection is the task of revealing inherent community structure. Since the networks can be either static or dynamic, community detection can be done on both static and dynamic networks as well. In this study, we have talked about taxonomy of community detection methods with their shortages. Then we examine and categorize application areas of community detection in the realm of nature of complex networks (i.e., static or dynamic) by including sub areas of criminology such as fraud detection, criminal identification, criminal activity detection and bot detection. This paper provides a hot review and quick start for researchers and developers in community detection area.
\end{abstract}

Index Terms - Complex networks, community detection, application of community detection.

\section{INTRODUCTION}

With digital era, we are all intimate with information systems. In the realm of today's world, information systems are represented by complex networks. One of the characteristics of complex networks is that they inherently contain a community structure.

Community structures observed in complex networks can be different in their natures such as disjoint, overlapping, hierarchical and local communities. Disjoint community structure includes communities with no overlap, which is illustrated in Fig 1. (a). That is, the members of this type of communities can be assigned to only one group. Overlapping community structure represents a structure that a member of any communities can have one or more membership of other communities, which is seen in Fig 1. (b). That is to say, a person can be members of different interest groups on an online social network at the same time. Hierarchical community structure shows hierarchical grouping levels as seen in Fig. 1. (c). As for local communities, they show different structure from local view, but no structure from global perspective, which is illustrated in Fig. 1. (d).

Since domain dependency and diversity in nature of communities on the given network is unknown beforehand, community definition is an ill-defined concept [1]. Even so, a general adopted definition of community according to structure of the network is that within community members are highly connected and across community members are loosely connected [2]. Communities can come into existence according to not only their structural similarities but also functional similarities among the members of the network [3]. Therefore, detecting community structure provides us meaningful insights about the network structure and its organization principle.

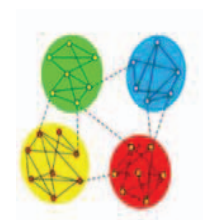

(a)

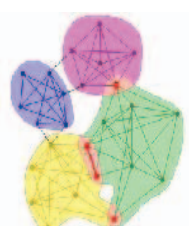

(b)

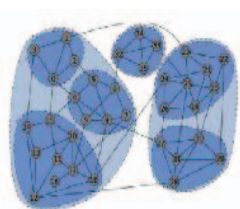

(c)

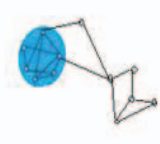

(d)
Figure 1. An example of illustrating different types of communities: (a) disjoint, (b) overlapping, (c) hierarchical and (d) local communities

Community detection is the task for revealing community structure of a given network at current time interval. It provides us a power to look from mesoscale (group-level) perspective. Therefore, it has many application areas where group-level tasks are done. It is used for market segmentation, criminal detection, recommendation systems and many more. For example, community detection is used in criminology by Pinheiro's work [4] by detecting anomalies on customer behaviors as possibly fraud. Another example for usage of community detection in criminology is done by Waskiewicz's work [5] to detect and reveal terrorist groups in terrorist social networks.

Since complex networks are modelled as either static or dynamic in its nature, community detection can be done for both. Static network may be considered as just frozen network for a specified time interval. However, communities in the network may growing or shrinking in size, even new communities may appear when some of them may disappear when time goes on. Dynamic community detection helps to detect and handle gracefully with this dynamicity. In summary, static community detection is interested in finding actual community structure as dynamic community detection is interested in detecting and tracking evolution of community structure over time.

In literature, there is a study [6] about community detection practical applications. However, it does neither give a taxonomy about the community detection methods and detailed explanation about them. It does not cover hot application areas as well. Additionally, it does not match application areas with network nature. To overcome this shortage, in this study, we (i) give a taxonomy of community detection methods, (ii) examine and categorize practical application areas of community detection according to their working nature (i.e., either static or 
dynamic) and (iii) talk about possible effects of some improvements on shortage of community detection methods on some case studies in criminology especially fraud detection, criminal identification, criminal activities detection and bot detection as well. This paper provides a hot review and quick start for researchers and developers in community detection.

The rest of the paper is organized as follows. In Section II, we give preliminary information about community structure, static community detection and dynamic community detection within a taxonomy with their shortages. Next, we present application areas of community detection in Section III. In Section IV, we close the paper with our closing thoughts that includes application areas, sub areas and network applicability of community detection method (i.e., static, dynamic or both applicable) and possible effects of improvements in community detection methods for criminology area.

\section{PRELIMINARIES}

In this part, we give a brief information about community structure, static and dynamic networks and static and dynamic community detection methods.

\section{A. Community Structure}

For a given network, represented by a graph $\mathrm{G}=\mathrm{V}, \mathrm{E}$ where $\mathrm{V}$ is the set of nodes and $\mathrm{E}$ the set of edges, the community detection task is interested in finding a community structure for a given graph (i.e., network). Community structure is the partition of the nodes in $\mathrm{V}$ of the form $\mathrm{C}=\mathrm{C}_{1}, \ldots, \mathrm{C}_{\mathrm{k}}$ such that each $\mathrm{C}_{\mathrm{i}}, 1 \leq \mathrm{i} \leq \mathrm{k}$ exhibits the community structure that presents groups of nodes so called communities .

Let's assume that we are given a social network as seen in Figure 2. If a static community detection algorithm is run on it, we can obtain a community structure as seen in Figure 3. That is, the network seen in Fig. 2 is partitioned into five sub groups (i.e., communities as $\mathrm{C}_{1}, \mathrm{C}_{2}$ etc.) as seen in Figure 3. It is worth to note that each community detection method does not have to be provide same partitioning. That is, you may or may not see the same communities seen in Fig. 3.

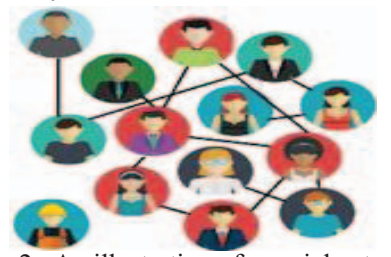

Fig. 2. An illustration of a social network

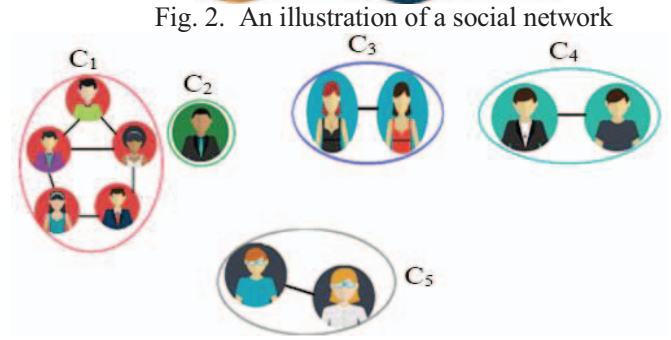

Figure 3. An illustration of a community detection algorithm application on the social network in Fig.2.

\section{B. Static Community Detection}

Graphs are good data structures to representing complex relationships. Therefore, community detection is done on complex network graphs that may be directed/undirected or weighted/unweighted or multiple-edges. In the context of this study, we examine all kind of graphs used by the works that we touch in the literature.

Static networks are regarded as snapshots of a network for a given time interval. As for static community detection, it tries to reveal underlying community structure by partitioning the network snapshot into different partitions according to friendship or relation properties or some properties of interests.

Many researchers have contributed in the field of static community detection on complex networks. Even if there are some categorizations in the literature, Fortunato [7] divides the traditional methods into four broad categories as spectral methods, methods based on statistical inference, methods based on optimization and methods based on dynamics.

(i) Spectral methods use spectral properties like eigen value spectrum of the matrix representations (e.g., adjacency matrix, Laplacian matrix, modularity matrix etc.) of networks to detect communities [1]. They generate a projection of vertices into a metric space by using eigen vectors as coordinates. For example, $\mathrm{i}^{\text {th }}$ entries of the eigen vector represent coordinates of vertex $\mathrm{i}$ in a m-dimensional Euclidean space where $\mathrm{m}$ is the number of eigen vectors used. Then, they apply a clustering algorithm like k-means to detect communities. However, they are not reliable when the network is very sparse because separation of eigenvalues is not sharp. They are computationally expensive as well. The works [8] [9] in can be given as examples of spectral methods.

(ii) Methods based on statistical inference generally adopts an ordinary approach to fit data to a generative network model. Most popular generative model with communities for networks is Stochastic Block Model (SBM). SBM depends on the maximization of log-likelihood of communities in the given graph. However, the methods using SBM need to know the number of communities in advance, which is unknown in real world networks in advance. The works in $[10,11]$ can be given as examples of statistical inference-based community detection methods.

(iii) Methods based on optimization try to find a maximum or minimum of a quality function that shows the quality of community structure. Most popular quality function is modularity proposed by Newman and Girvan [12]. Modularity is a concept depending on the maximization of difference between actual network and another form of actual network that have randomly destroyed community structure. Modularity maximization is an NP-hard problem [13]; therefore, approximation algorithms or heuristics are used. Modularity may be not detecting small size communities (i.e., resolution limit) as well. Additionally, they can suffer from instability problem (i.e., on each run they may detect different communities even on the same network). The methods in $[14,15]$ can be given as examples of methods based on optimization.

(iv) Methods based on dynamics uses running dynamics of the networks like diffusion, random walk and spin dynamics to detect community structure in a network. The methods using label propagation technique is based on 
diffusion process. Most popular dynamic are random walk dynamics. The methods using random walks using homophily assumption. That is, vertices in similar nature is highly probable in same partition. Methods using spin dynamics first define a spin model on the network where spin variables are assigned to vertices of the network. Then, they assign an energy of the spin via Hamiltonian distance. They aim to find spin configurations that give minimum Hamiltonian distance. The works in $[16,17]$ can be given as examples for methods based on dynamics.

\section{Dynamic Community Detection}

We give a brief description for each community evolution approaches we have identified in the literature, framing them in the taxonomy in [18]. The taxonomy is a three-level classification of existing methods for tracking community evolution in dynamic social networks with respect to their network models (first level of tree structure), their functioning principles (second level) and algorithmic techniques (third level). According to their functioning principles, there are four fundamental approaches:

(i) Independent Community Detection and Matching contains methods that first detect community structure on each snapshot separately and match those communities across consecutive or non-consecutive time-stamps. All methods in this category target to track community evolution by identifying key community events (e.g., birth, death, growth etc.) through life cycles of communities. Core-based methods identify one or several specific nodes for each community called as core nodes. For example, the nodes have highest centrality value on the network can be core nodes. Then, the methods determine community events according to the core nodes. As for event-based methods, they consider all nodes to determine the community events. The works in [19-21] can be given as examples of dynamic community detection methods that use this approach.

(ii) Dependent Community Detection covers methods that detect community structure on snapshot at time $t$ and past community information (i.e., on previous snapshot or some recent snapshots). In this approach, broadly there are two types of methods: evolutionary methods and costfunction methods. Evolutionary methods built on or modify The methods using this approach are based on coupling graphs or either optimizing metrics. In coupling graphbased methods, the basic idea is to build a coupling graph and detect communities on this graph. In metric optimization-based methods, the basic idea is designing a metric that can be directly optimized on all snapshots given. The works in $[25,26]$ can be given as examples of dynamic community detection that use this approach.

(iv) Dynamic Community Detection on Temporal Networks includes methods that detect community on only first snapshot network, then alter this community structure for each incoming update. The works in [27, 28] can be given as examples of dynamic community detection that use this approach. basic community detection algorithms like Louvain [22]. They initialize community structure with this algorithms and re-run modified basic algorithm again. As for cost-function methods, they use a cost-function (i.e., any function to minimize the community changes between successive snapshots such as Modularity maximization between two consequent snapshots). The works in [23, 24] can be given as examples of dynamic community detection that use this approach.

(iii) Simultaneous Community Detection on All Snapshots encloses methods that first construct a single from all snapshots so-called coupling graph and detect community structure on the coupling (joint) graph.

Table 1. Community Detection methods and their shortages

\begin{tabular}{|c|c|c|}
\hline $\begin{array}{c}\text { Community } \\
\text { Detection Methods }\end{array}$ & $\begin{array}{c}\text { Network } \\
\text { Type }\end{array}$ & Shortage/Drawbacks \\
\hline Spectral methods & Static & $\begin{array}{l}\text {-Not computationally efficient } \\
\text {-Not reliable for sparse networks }\end{array}$ \\
\hline $\begin{array}{l}\text { Methods based on } \\
\text { statistical inference }\end{array}$ & Static & $\begin{array}{l}\text {-Selection of models } \\
\text {-High time complexity } \\
\text {-Specifying community numbers } \\
\text { in advance. }\end{array}$ \\
\hline $\begin{array}{l}\text { Methods based on } \\
\text { optimization }\end{array}$ & Static & $\begin{array}{l}\text {-Resolution Problems } \\
\text {-Instability Problems }\end{array}$ \\
\hline $\begin{array}{l}\text { Methods based on } \\
\text { dynamics }\end{array}$ & Static & $\begin{array}{l}\text {-They may need other clustering } \\
\text { algorithms to work } \\
\text {-They may suffer from poor } \\
\text { stability }\end{array}$ \\
\hline $\begin{array}{l}\text { Independent } \\
\text { community detection } \\
\& \text { matching }\end{array}$ & Dynamic & $\begin{array}{l}\text {-High time complexity } \\
\text {-Instability of traditional } \\
\text { community detection methods }\end{array}$ \\
\hline $\begin{array}{l}\text { Dependent } \\
\text { community detection }\end{array}$ & Dynamic & $\begin{array}{l}\text {-No chance to parallelize } \\
\text { community detection on } \\
\text { snapshots } \\
\text {-Traditional community } \\
\text { detection methods are not } \\
\text { directly applicable }\end{array}$ \\
\hline $\begin{array}{l}\text { Simultaneous } \\
\text { community detection } \\
\text { on all snapshots }\end{array}$ & Dynamic & $\begin{array}{l}\text {-There is no chance to update the } \\
\text { network structure. } \\
\text {-Difficulty in handling some } \\
\text { operations such as merge and } \\
\text { split }\end{array}$ \\
\hline $\begin{array}{l}\text { Dynamic community } \\
\text { detection on temporal } \\
\text { networks }\end{array}$ & Dynamic & $\begin{array}{l}\text {-Community drift can be } \\
\text { occurred } \\
\text {-Traditional community } \\
\text { detection methods are not } \\
\text { directly applicable }\end{array}$ \\
\hline
\end{tabular}

In Table 1, community detection methods and their shortages /drawbacks are seen.

\section{PRACTICAL APPLICATIONS OF COMMUNITY DETECTION}

Community detection is worked by many researchers from different disciplines so far. This paper represents such practical applications by categorizing them according to their domains rather than just chronological order.

\section{A. Criminology}

Community detection is used for identification of criminal user groups. Those groups can be built from either real person accounts or bot accounts. They can support or diffuse criminal ideas or terrorism-like activities. The authors in work [29] use community detection to detect communities in criminal networks, then they do some 
manual analysis. Pinheiro [4] make a study to identify fraud events on telecommunication networks by using community detection that helps to determine customer behaviors and examining outliers as possibly fraud. Similarly, Waskiewicz [5] provide a study to detect terrorist group activities on some online social networks by using community detection to detect such groups.

Additionally, usually bots(i.e., software robots) popularly used by attackers for impersonation for identity frauds, follower frauds and botnet attacks. The works for bot detection via community detection is introduced in detail in $[30]$.

\section{B. Public Health}

In health domain, community detection is generally used for discovering dynamics of certain groups susceptible to an epidemic disease. Salathe and Jones[31] show the impact of community structure on disease dynamics.

Community detection is used detect a disease such as cancer and tumor types as well. Bechtel et al.[32] proposed a community-based lung cancer detection approach. Likewise, Haq and Wang [33] make a study on genomic datasets for detecting subgroups of twelve types of cancers and they look survival rates of those communities and distribution of tumor types over communities.

Additionally, community detection is used to organ detection. The works in $[34,35]$ are examples for organ or tissue detection.

\section{Politics}

In Politics, community detection is used for observation of influences of political ideologies or individual politicians on some social group. Later, it can be specialized to track evolution of this influence over time. This influence commonly is created by influence or astroturfer bots - bots make an attempt to create a fake impression on real grassroots to support a policy, individual, product campaign [36]. Community detection is used for detecting those types of bots as well [30].

\section{Customer Segmentation, Smart Advertising and Targeted Marketing}

Community detection is used for customer segmentation directly, smart advertising and targeted marketing indirectly by companies as well. Companies can provide better service solution if they know their customer groups intimately(i.e., customer segmentation). Then, they can do advertising and marketing for detected specific groups [37].

\section{E. Recommendation Systems}

Recommendation systems are another important services we use everyday whenever we wanto to buy a book from a website, watch a video or listen a music on a social media site etc. They try to recommend something that probably you would like to glad to meet. The task of community detection is akind of segregation of people which is like-mind. Although there are many works in the literature that use community detection in recommendation systems, we give only some example studies in $[38,39]$.

\section{F. Social Network Analysis}

Community detection can be a good means to understand communities on networking level and correlate them in real-life relations. For example, community detection on social networks and online social networks such as Facebook, Twitter, LinkedIn etc. are good examples for this area.

Social Network Analysis is one of the mostly used community detection; therefore, there are many works in the literature. Some of them are given in [40, 41].

\section{G. Network Summarization and Privacy}

Community detection provide a mesoscale view of networks. That is, it is provide a group-level point of view and summarize the network in group level. Therefore, it can help serving privacy in the network when sharing generalized features of them with another party.

On the other hand, community detection can be used break privacy of people on weak signal networks like Bitcoin. Authors of the work [42] used community detection to show how it can be efficiently used to reidentify multiple addresses that belong to same user. Fortunately, Kokkiligadda and Vatsavayi [43] and the authors of the work [44] show that anonymized social networks satisfactorily preserve the community structure of their original networks. That is, except weak signal networks, community detection does not lead to privacy leakage.

\section{H. Link Prediction}

Link prediction assess the possibility of future links between members of a network and it is used for determining fake links, missing links and future links. The underlying structure of the network is found via a community detection algorithm, then the possibility of being a link between two members is calculated. ValverdeRebaza and Lopes [45] propose an approach based on community detection for link prediction. Similarly, Soundarajan and Hopcroft [46] show that community information obtained by community detection methods increase the accuracy of similarity-based link prediction.

\section{Community Evolution Prediction}

Community evolution prediction regards the prediction of future form of a community given its past and present form in terms of community events such as growing, shrinking, merging, forming, solving etc. It is one of the hot topics in community analysis field. Naturally, there are some works in literature. Those address the predictability of community evolution issue as supervised learning task. Some of them like Brodka, Kazienko and Kołoszczyk[47] employ ordinary classifiers (e.g., Naïve Bayes, Bayes Net, Logistic Regression, SVM etc.) whereas others like the authors of [48] use both sequential (i.e., Conditional Random Fields with Linear 
Chain and with Skip Chain) and ordinary classifiers for prediction. To achieve community evolution prediction, a dynamic community detection is needed to see evolution of the communities.

\section{CONCLUSION}

From mentioned application areas just above, community detection has wide range of application domains. Some of them use static community detection, some other dynamic community detection or both. In Table 2, areas and usable community detection method categories are shown.

The number of applications presented here is neither complete nor exhaustive. However, we presented only a bunch of sample applications that indicate the usefulness of community detection. In future, it can be applied to smart cities or any other emerging fields that working on a group-level tasks; therefore, it should not be underestimated.

Let's focus the works in criminology area in Table 2. If there is an improvement on shortages mentioned in Table 1, there will be some effects on these works as well.

Table 2. Application areas, sub-areas and nature of correspondent community detection method

\begin{tabular}{|l|l|l|}
\hline \multirow{4}{*}{ Application Area } & Sub-Area & $\begin{array}{l}\text { C.D. } \\
\text { Method }\end{array}$ \\
\hline \multirow{4}{*}{ Priminology } & Criminal Identification & Static \\
\cline { 2 - 3 } & Fraud Detection & Static \\
\cline { 2 - 3 } & Criminal Activities Detection & Static \\
\cline { 2 - 3 } & Bot Detection & Static \\
\hline \multirow{2}{*}{ Politics } & $\begin{array}{l}\text { Dynamics of Epidemic } \\
\text { spreading }\end{array}$ & Dynamic \\
\cline { 2 - 3 } & Cancer/Tumor Detection & Static \\
\cline { 2 - 3 } & Tissue/Organ Detection & Static \\
\hline Smart Advertising & Evolution of Influence & Dynamic \\
\cline { 2 - 3 } Targeted Marketing & Astroturfing & Static \\
\hline $\begin{array}{l}\text { Recommendation } \\
\text { Systems }\end{array}$ & Customer Segmentation & Static \\
\hline $\begin{array}{l}\text { Social Network } \\
\text { Analysis }\end{array}$ & Customer Segmentation & Static \\
\hline $\begin{array}{l}\text { Network } \\
\text { Summarization }\end{array}$ & Community Detection & Static \\
\hline Privacy & Group Segmentation & Both \\
\hline Link Prediction & Link Prediction & Static \\
\hline $\begin{array}{l}\text { Pommunity Evolution } \\
\text { Predion }\end{array}$ & Community Evolution \\
\hline
\end{tabular}

For example, the work in [29] use a method based on optimization and it suffers from instability problem. If instability problem is solved for the method, it produces more stable community structure and analysis done on this structure will be more coherent and reliable. Similarly, the community structures detected by other works $[4,5]$ will be more dependable, and produce better results.

In this study, we introduce a taxonomy of community detection methods and share their shortages, which is lead to possible research areas. We discussed possible effects of possible improvements of instability of community detection algorithms on some case studies on criminology area. Then, we create a summary table that contains application areas and corresponding community detection method type. By using this table, researchers and developers can do a quick-start in the field.

\section{ACKNOWLEDGMENT}

This work is partially supported by YOK through its 100/2000 and TÜBİTAK through 2011-C PhD Scholarship Programmes to whom we are most grateful.

\section{REFERENCES}

[1] S. Fortunato, "Community detection in graphs," Physics Rep., vol. 486, no. 3-5, pp. 75-174, 2010.

[2] M. Girvan, and M. E. Newman, "Community structure in social and biological networks," Proc. Nat. Academy of Sci., vol. 99, no. 12, pp. 7821-7826, 2002.

[3] M. E. Newman, "Detecting community structure in networks," The European Physical J. B., vol. 38, no. 2, pp. 321-330, 2004.

[4] C. A. R. Pinheiro, "Community detection to identify fraud events in telecommunications networks," SAS SUGI proceedings: customer intelligence, 2012.

[5] T. Waskiewicz, "Friend of a friend influence in terrorist social networks," in Proc Int. Conf. on Artificial Intelligence, 2012, p. 1.

[6] M. Ahuja, and J. Singh, Neha, "Practical applications of community detection," Int. J. Advanced Research in Comput. Sci. and Software Eng.vol. 6, no. 4, pp. 412-415,2016.

[7] S. Fortunato, and D. Hric, "Community detection in networks: A user guide," Physics Rep., vol. 659, pp. 1-44, 2016.

[8] M. E. Newman, "Finding community structure in networks using the eigenvectors of matrices," Physical Review E, vol. 74, no. 3, pp. 036104, 2006.

[9] M. E. Newman, "Spectral methods for community detection and graph partitioning," Physical Review E, vol. 88, no. 4, pp. 042822, 2013.

[10]E. Côme, and P. Latouche, "Model selection and clustering in stochastic block models based on the exact integrated complete data likelihood," Statistical Modelling, vol. 15, no. 6, pp. 564-589, 2015.

[11]M. E. Newman, and G. Reinert, "Estimating the number of communities in a network," Physical Review Lett., vol. 117, no. 7, pp. 078301, 2016

[12] M. E. Newman, and M. Girvan, "Finding and evaluating community structure in networks," Physical Review E, vol. 69, no. 2, pp. 026113, 2004.

[13]M. Chen, K. Kuzmin, and B. K. Szymanski, "Community detection via maximization of modularity and its variants," IEEE Trans. Comput. Social Syst., vol. 1, no. 1, pp. 46-65, 2014

[14]A. Clauset, "Finding local community structure in networks," Physical Review E, vol. 72, no. 2, pp. 026132, 2005.

[15]A. Lancichinetti, and S. Fortunato, "Limits of modularity maximization in community detection," Physical Review E, vol. 84, no. 6, pp. 066122, 2011.

[16] J. Reichardt, and S. Bornholdt, "Statistical mechanics of community detection," Physical Review E, vol. 74, no. 1, pp. 016110, 2006.

[17]M. Rosvall, and C. T. Bergstrom, "Maps of random walks on complex networks reveal community structure," Proc. Nat. Academy of Sci., vol. 105, no. 4, pp. 1118-1123, 2008.

[18]N. Dakiche, F. B.-S. Tayeb, Y. Slimani, and K. Benatchba, "Tracking community evolution in social networks: A survey," Information Processing \& Management, 2018 [Online] Available: https://www.sciencedirect.com/science/article/pii/S03064573173055 $\underline{51}$

[19] D. Greene, D. Doyle, and P. Cunningham, "Tracking the evolution of communities in dynamic social networks," in Proc. Int. Conf. on Advances in social networks analysis and mining, 2010, pp. 176183.

[20] J. Hopcroft, O. Khan, B. Kulis, and B. Selman, "Tracking evolving communities in large linked networks," Proc. Nat. Academy of Sci., vol. 101, no. suppl 1, pp. 5249-5253, 2004.

[21]E. G. Tajeuna, M. Bouguessa, and S. Wang, "Tracking Communities over Time in Dynamic Social Network," Machine Learning and Data Mining in Pattern Recognition, , vol 9729, pp. 341-345, Perner P, Eds: Springer, 2016.

[22] V. D. Blondel, J.-L. Guillaume, R. Lambiotte, and E. Lefebvre, "Fast unfolding of communities in large networks," J. statistical mechanics: theory and experiment, vol. 2008, no. 10, pp. P10008, 2008. 
[23]J. Sun, C. Faloutsos, S. Papadimitriou, and P. S. Yu, "Graphscope: parameter-free mining of large time-evolving graphs," in Proc. 13th ACM SIGKDD Int. Conf. on Knowledge discovery and data mining, 2007, pp. 687-696.

[24] J. Zhu, J. Liu, X. Zhang, and Y. Zhao, "A reconstructed event-based framework for analyzing community evolution," in 2016 IEEE Int. Conf. on Big Data Analysis, 2016, pp. 1-4.

[25] B. Mitra, L. Tabourier, and C. Roth, "Intrinsically dynamic network communities," Comput. Networks, vol. 56, no. 3, pp. 1041-1053, 2012.

[26]P. J. Mucha, T. Richardson, K. Macon, M. A. Porter, and J.-P. Onnela, "Community structure in time-dependent, multiscale, and multiplex networks," Sci., vol. 328, no. 5980, pp. 876-878, 2010.

[27] G. Rossetti, L. Pappalardo, D. Pedreschi, and F. Giannotti, "Tiles: an online algorithm for community discovery in dynamic social networks," Machine Learning, vol. 106, no. 8, pp. 1213-1241, 2017.

[28] A. Zakrzewska, and D. A. Bader, "Tracking local communities in streaming graphs with a dynamic algorithm," Social Network Analysis and Mining, vol. 6, no. 1, pp. 65, 2016.

[29] H. Sarvari, E. Abozinadah, A. Mbaziira, and D. Mccoy, "Constructing and analyzing criminal networks," in 2014 IEEE Security and Privacy Workshops , 2014, pp. 84-91.

[30] A. Karataş, and S. Şahin, "A Review on Social Bot Detection Techniques and Research Directions," in Proc. Int. Security and Cryptology Conference Turkey, 2017, pp.156-161.

[31] M. Salathé, and J. H. Jones, "Dynamics and control of diseases in networks with community structure," PLoS Computational Biology, vol. 6, no. 4, pp. e1000736, 2010.

[32] J. J. Bechtel, W. A. Kelley, T. A. Coons, M. G. Klein, D. D. Slagel, and T. L. Petty, "Lung cancer detection in patients with airflow obstruction identified in a primary care outpatient practice," Chest, vol. 127 , no. 4, pp. 1140-1145, 2005.

[33]N. Haq, and Z. J. Wang, "Community detection from genomic datasets across human cancers" 2016 IEEE Global Conf. on Signal and Infor. Process., 2016,pp. 1147-1150.

[34] F. Taya, J. de Souza, N. V. Thakor, and A. Bezerianos, "Comparison method for community detection on brain networks from neuroimaging data," Appl. Network Sci., vol. 1, no. 1, pp. 8, 2016.

[35] Y. Yang, P. G. Sun, X. Hu, and Z. J. Li, "Closed walks for community detection," Physica A: Statistical Mechanics and its Applications, vol. 397, pp. 129-143, 2014.

[36]A. Bienkov, "Astroturfing: what is it and why does it matter?," [Online]. Available: https://www.theguardian.com/commentisfree/2012/feb/08/what-isastroturfing

[37] M. J. Mosadegh, and M. Behboudi, "Using social network paradigm for developing a conceptual framework in CRM," Australian J. Bus. and Manage. Research, vol. 1, no. 4, pp. 63, 2011.

[38] S. B. Abdrabbah, R. Ayachi, and N. B. Amor, "Collaborative filtering based on dynamic community detection," Dynamic Networks and Knowledge Discovery, vol. 85, 2014.

[39] D. Lalwani, D. V. Somayajulu, and P. R. Krishna, "A community driven social recommendation system," in Proc. 2015 IEEE Int. Conf. on Big Data, 2015, pp. 821-826.

[40] Y. Atay, I. Koc, I. Babaoglu, and H. Kodaz, "Community detection from biological and social networks: A comparative analysis of metaheuristic algorithms," Appl. Soft Computing, vol. 50, pp. 194$211,2017$.

[41] C. Wang, W. Tang, B. Sun, J. Fang, and Y. Wang, "Review on community detection algorithms in social networks," in Proc. IEEE Int. Conf. on Progress in Informatics and Computing,2015, pp. 551555 .

[42] C. Remy, B. Rym, and L. Matthieu, "Tracking bitcoin users activity using community detection on a network of weak signals," in Int. Workshop on Complex Networks and their Applications, 2017,pp. 166-177.

[43]N. Kokkiligadda, and V. K. Vatsavayi, "Community privacy preservation in dynamic social networks.", Int. J. Tech. Research and Applicat., vol. 4, no.6 , pp. 133-136, 2016.

[44] Campan, Y. Alufaisan, and T. M. Truta, "Community Detection in Anonymized Social Networks," in Proc. Workshops of the EDBT/ICDT 2014 Joint Conference, 2014, pp. 396-405.

[45] J. C. Valverde-Rebaza, and A. de Andrade Lopes, "Link prediction in complex networks based on cluster information," Proc. $21^{\text {st }}$ Brazilian Conf. in Artificial Intelligence,2012, pp. 92-101.
[46] S. Soundarajan, and J. Hopcroft, "Using community information to improve the precision of link prediction methods," in Proc. 21st Int. Conf. on World Wide Web,2012, pp. 607-608.

[47]P. Bródka, P. Kazienko, and B. Kołoszczyk, "Predicting group evolution in the social network," in Int. Conf. on Social Informatics, 2012, pp. 54-67.

[48] G. Diakidis, D. Karna, D. Fasarakis-Hilliard, D. Vogiatzis, and G. Paliouras, "Predicting the evolution of communities in social networks," in Proc. 5th Int. Conf. on Web Intelligence, Mining and Semantics, 2015, p. 1. 